\title{
Withering away, weakly
}

\section{F. A. Muller}

Received: 15 October 2008 / Accepted: 1 April 2009 / Published online: 11 August 2009

(c) The Author(s) 2009. This article is published with open access at Springerlink.com

\begin{abstract}
One of the reasons provided for the shift away from an ontology for physical reality of material objects \& properties towards one of physical structures \& relations (Ontological Structural Realism: OntSR) is that the quantum-mechanical description of composite physical systems of similar elementary particles entails they are indiscernible. As material objects, they 'whither away', and when they wither away, structures emerge in their stead. We inquire into the question whether recent results establishing the weak discernibility of elementary particles pose a threat for this quantum-mechanical reason for OntSR, because precisely their newly discovered discernibility prevents them from 'whithering away'. We argue there is a straightforward manner to consider the recent results as a reason in favour of OntSR rather than against it.
\end{abstract}

Keywords Quantum mechanics · Structural realism · Identical particles

\section{Whither elementary particles?}

According to our best scientifically informed philosophical views, everything in the universe, from the body of Albert Einstein to the brain of Angelina Jolie and from the music of Friedrich Nietzsche to the ideas of Igor Strawinsky, ultimately either

\footnotetext{
F. A. Muller $(\varangle)$

Faculty of Philosophy, Erasmus University Rotterdam, Burg. Oudlaan 50, 3062 PA, Rotterdam, The Netherlands

e-mail: f.a.muller@uu.nl

F. A. Muller

Department of Physics and Astronomy, Utrecht University, Budapestlaan 6, IGG-3.08, 3584 CD, Utrecht, The Netherlands
} 
consists of or supervenes on pieces of interacting matter. Without interacting matter there would be nothing, perhaps not even space-time. According to our best scientific theories, every piece of interacting matter in the universe is composed of elementary particles, ultimately quarks, leptons and gauge bosons. Hence everything in the universe ultimately either consist of or supervenes on elementary particles. This makes elementary particles truly and uniquely fundamental.

According to quantum mechanics (QM), elementary particles are unlike tiny indivisible pieces of rigid matter in motion, occupying a definite location at every instant of time, colliding with other particles. This much is pretty certain. In good Popperian spirit, we definitely know what an elementary particle is not.

But, then, what are elementary particles? What are they like? We want to know this.

QM tells us more, if only a little: elementary particles possess some properties, such as mass, charge and magnitude of spin. All other properties are however denied to them, unless we perform measurements on them. Just after measurements are performed on them, in the laboratory, they acquire some additional properties. But only for a split second. After measurements have ended, the properties pop out of existence again. And they pop back into existence upon measurement and only upon measurement, unpredictably. Elementary particles are somewhat like colourless, amorphous entities in a box that obtain a shining colour and assume a definite shape as soon as someone opens the box. Sheer magic. Real magic.

Elementary particles never occupy any definite location. They are neither somewhere nor everywhere. Yet there is a non-vanishing probability to find them anywhere upon measurement, also outside boxes with the thickest of walls, just after you put them in there and closed the box firmly. Elementary particles behave as if they were tiny billiard balls in some respects when they find themselves in certain particular circumstances, created by us; and they behave as if they were tiny waves in some respects when they find themselves in other particular circumstances, also created by us. This is called 'the wave-particle duality'. But, as Feynman knew, to introduce a word for something does not mean you understand it. They are like little schizophrenics it seems. The proposal to call them 'wavicles' rather than 'particles' to express this has not caught on, as have Nicholas Maxwell's proposal to call them 'propensitons' and Lévy-Leblond's to call them 'quantons'. Admittedly naming is not the same as understanding, but a different word just to signal that the quantum-mechanical particle-concept is very unlike the classical particle-concept we once knew, used and loved is a commendable idea.

When sets of what Dirac called similar elementary particles are considered (i.e. having the same few properties they do possess), they loose their individuality, as a consequence of the symmetrisation postulate of QM, according to which permuting them does not yield a situation that is physically discernible from the unpermuted one. Ever since Weyl (1928) brought it up about eighty years ago and Margenau (1949) emphasised it about twenty years later, philosophers of physics have been arguing that this means that QM refutes Leibniz's venerable principle of the identity of indiscernibles (PII); for when we can have composite physical systems consisting of indiscernible particles, PII is refuted because PII precisely forbids the existence of such indiscernible objects. So elementary particles are indiscernibles, precisely as 
Schrödinger has begged us to believe. ${ }^{1}$ After having taken cognizance of this, Quine overreacted by concluding that "matter evidently goes by the board".

When we take Einstein's insights into the nature of space and time into account, and wed QM to his Special Theory of Relativity by cutting away infinities that arise in the process (renormalisation), particles no longer live eternally. They can be annihilated and created. We can do it and we do it, in what Casimir has called 'the cathedrals of the twentieth Century': the high-energy particle accelerators that have the size of cities, those magnificent symbols of our pursuit to unravel the mysteries of the cosmos. By the way, the effect named after Casimir showed that quantum-physical empty space (the vacuum), i.e. without 'particles', is not a void but a plenum, seething with fluctuations; when you accelerate a 'particle-detector' through empty space, it registers something, as Unruh first demonstrated—presumably it detects so-called Rindler quanta. ${ }^{3}$ Long before talk of virtual reality became common, virtual elementary particles swarmed the stage of physics, in a limbo between definite existence and definite non-existence that still no one understands. And when elementary particles are localised somewhere, in space, at some point in time, upon measurement in a laboratory, they are not localised anywhere according to someone who happens to drive by the laboratory on her bicycle-for localisation is not Lorentz-invariant, as Wigner pointed out long ago. Bafflingly, a quantum theory of localisable particles in Minkowski space-time turns out to be mathematically impossible altogether, as Malament demonstrated. ${ }^{4}$ The marriage between QM and the Special Theory of Relativity seems the death-knell of the already meagre quantum-mechanical concept of a particle.

If these quantum-physical considerations do not make a case for a drastic revision of our fundamental ontology of material objects with properties, then we may safely predict that nothing will ever make such a case. ${ }^{5}$ At the truly and uniquely fundamental level of physical reality, there are no individual material objects with properties and relations that derive from properties. There are no individuals. There are no absolute discernibles. Let's face it, indiscernible objects are no objects at all. Identity conditions break down. No identity, no entity. Quantification breaks down. We can talk no more-and if someone wants to keep talking of physical objects nonetheless, then he must kiss standard mathematics and classical logic goodbye, must embrace an entirely different set-theory and logic, as Dalla Chiara, Di Franca, French and others have known for a while. ${ }^{6}$

All in all, elementary particles as material objects whither away. For whom science matters, every thing must go. What will replace them?

\footnotetext{
1 Weyl (1928, IV.C. § 9), Margenau (1949), Schrödinger (1996, p. 122), French (1989), French and Redhead (1988), Redhead and Teller (1992), Castellani and Mittelstaedt (2000), French and Ladyman (2003), French (2006).

2 Quine (1976b, p. 499).

3 See Redhead (1995).

${ }^{4}$ See Malament (1992), Halvorson and Clifton (2002).

5 We therefore disagree with Chakravartty (2003), who dares to claim that the quantum-physical considerations provide insufficient ground for rejecting a fundamental ontology of material objects and properties.

${ }^{6}$ See their contributions in Castellani (1998).
} 
Enter ontological structural realism. Elementary particles are structures. At the truly and uniquely fundamental level of physical reality there are only structures. Structures drive on relations. If structures have properties, these properties derive from relations. 'Particles' are no more than placeholders in structures. They don't really exist. Only structures exist. Schrödinger: "Some philosophers of the past, if the case could be put to them, would say that the modern atom consists of no stuff at all but is pure shape." 7

So the ontological structural realist lives happily ever after? Not so fast. Recently his happiness has come under threat. But before we expound the threat (Sect. 3) and try to allay it (Sect. 4), we first need to get clear on the relation between scientific realism and the metaphysical thesis of ontological structural realism, which we just introduced in passing (Sect. 2).

\section{Scientific structural realism}

When J. Ladyman introduced the distinction between Epistemic Structural Realism (EpSR) and Ontic Structural Realism (OntSR), and argued for OntSR, he called quantum mechanics (QM) to the witness-bench; other structural realists have endorsed this strategy, notably S. French. ${ }^{8}$ We briefly inquire into their relation to the general view of scientific realism, arguably still the dominant position in the realism debate in the philosophy of science. Here is a standard formulation of scientific realism.

Scientific Realism (ScR) Most of the posits of our theoretical scientific knowledge (objects, fields, structures, events, processes), unobservable ones notably included, exist human-independently in the actual world; these posits possess human-independently most of the properties ascribed to them by our theoretical scientific knowledge, and mutatis mutandis for the relations between them.

By 'theoretical scientific knowledge' we mean the propositional content of all well-established scientific theories and models. ${ }^{9}$ The presence of the adverb 'humanindependently' serves to emphasise that scientific realism (ScR), being realism with regard to science, stands opposed to idealism. Whatever posits with their properties and relations that the realist claims there are, they are there independent of our existence and our activities and whatever else comes along with that. The mentioning of unobservables in ScR merely serves to emphasise that they (or most of them) are included: 'Most' in ScR does not mean a restriction to observables only. ${ }^{10}$ Furthermore, the occurrence of 'the actual world' signals explicitly that ScR, as formulated here, does not is comprise modal claims.

\footnotetext{
7 Schrödinger in Castellani (1998, p. 203).

8 Ladyman (1998), French and Ladyman (2003).

9 Other kinds of scientific knowledge are not included here, such as how to conduct an experiment, how to test an hypothesis, and how to apply knowledge for the benefit of mankind, because they do not bear on the issue at hand.

10 For what unobservables are, see Monton and van Fraassen (2003), and Muller (2005).
} 
Roughly summarised, ScR says that science provides us with knowledge of the actual world. A more restrictive and therefore more modest realist claim is the following one:

Scientific Epistemic Structural Realism (ScEpSR) All our theoretical scientific knowledge of the actual physical world is knowledge of its structures.

The restriction is two-fold: to structures and to the physical world. The last-mentioned restriction leaves room for mental reality; it leaves room for a reduction of the mental to the physical, for supervenience of the mental on the physical, and even for a Cartesian substance dualism. ScEpSR makes no pronouncements on mentality and does not harbour any kind of (anti-)physicalism. For instance, the existence of mental non-structural entities is compatible with ScEpSR.

Further, debates on and elaborations of structural realism generally have been focussing on physics, and not, for instance, on paleontology, zoology or astronomy, to mention a few branches of natural science only. This is understandable, because ScEpSR was intended by its modern originator, J. Worrall, as being 'the best of both worlds', in that it was supposed to be a metaphysically meagre realism so as not to fall prey to the pessimistic meta-induction over the history of science, but still a sufficiently muscular realism to provide success-arguments in its defence (e.g. Putnam's 'no-miracle argument'). ${ }^{11}$ Since the pessimistic meta-induction thrives on the history of physics, rather than on the history of paleontology, zoology or astronomy, the debate has therefore focussed on physics. We soon join in.

When knowledge implies truth, as few epistemologists would care to deny, then ScEpSR implies that all and only propositions of our theoretical scientific knowledge that concern structures are true. Hence the physical world consists of structures, which exist human-independently because science does not connect the relevant existenceclaims to our existence or our activities - this is almost but not yet ontological structural realism (read on). Does the physical world consist only of structures? Let us also adopt

Scientific Optimism (ScOpt) Science will, in the end, provide us with everything that we, humans, can come to know about the physical world.

When ScEpSR implies that the physical world consists of structures, then in combination with $\mathrm{ScOpt}$ we arrive at

Ontological Structural Realism (OntSR) The physical world consists of structures only and they exist human-independently.

Conversely, if structures are all there is in the physical world (OntSR), then all we can come to know of the physical world by scientific inquiry is knowledge of structures. All theoretical scientific knowledge that we actually posses then must be knowledge of structures only; this is ScEpSR.

$\overline{11 \text { Worrall (1989), who focussed }}$ on nineteenth-century physics. 
To summarise, we have just argued for the following

$$
(\mathrm{ScOpt} \wedge \mathrm{ScEpSR}) \longrightarrow \text { OntSR and OntSR } \longrightarrow \text { ScEpSR, }
$$

from which it follows that for scientific optimists the difference between OntSR and ScEpSR is of little significance:

$$
\mathrm{ScOpt} \longrightarrow(\mathrm{OntSR} \longleftrightarrow \mathrm{ScEpSR})
$$

Hence debates amongst optimistic scientific realists whether an ontological or an epistemological variant of ScR should be adopted are without substance. Substance would return if ScOpt were rejected, but even then adherence to OntSR would still imply adherence to ScEpSR. Only ScEpSR would then no longer imply OntSR, although also in that case it would follow that the physical world consists of structures as far as science can tell, but that, then, there is more between heaven and earth than dreamt of in science and what is beyond these dreams and inhabits the physical world may be non-structural in nature. Whether such a rejection of ScOpt is scientific pessimism or anti-scientific obscurantism is an issue we need not decide for the purposes of the current paper.

Recall that the conclusion of the previous Section was that quantum physics, which is part of our theoretical scientific knowledge, is telling us that elementary particles are not material objects but structures. This is ScEpSR; and for the scientific optimist we then have OntSR by virtue of (1). But at the end of the previous Section we also mentioned a threat to the claim that elementary particles are not material objects, which eo ipso constitutes a threat to both ScEpSR and OntSR. We now turn to the source of this threat.

\section{Weak discernibles}

Recently it has been argued that similar particles can be saved from indiscernibility. ${ }^{12}$ They can be discerned by physically meaningful binary relations that are permutation-invariant; that is, by relations that are symmetric and either irreflexive or reflexive. This makes the particles weakly discernible, to follow Quine's terminology. ${ }^{13}$ What is more, these relations are categorical in that they do not involve the quantum-mechanical probabilities. Call an object (in a set $S$ of objects) that is absolutely discernible - meaning that it has a property that no other object in $S$ has-an individual (so that its 'individuality' resides in that property); call an object that is relationally discernible - meaning that it is discerned from all other objects in $S$ by a relation-but not absolutely discernible a relational; and call an object that is neither an individual nor a relational indiscernible. ${ }^{14}$ Then elementary particles are not indiscernibles but

\footnotetext{
12 Saunders (2006), Muller and Saunders (2008), Muller and Seevinck (2009).

13 Quine (1976a).

14 See Muller and Saunders (2008, pp. 503-504) for this terminology.
} 
relationals. We provide two examples of this and refer to the relevant literature for the general theorems.

Example 1 Consider a composite physical system of two fermions having spin $\hbar / 2$ and finite-dimensional Hilbert-space $\mathcal{H}=\mathbb{C}^{2}$; then $\mathbb{C}^{2} \otimes \mathbb{C}^{2}$ is the pure state space of the composite system. We have a set $\{\boldsymbol{1}, 2\}$ of two particles, having names (or labels) ' $\boldsymbol{l}$ ' and ' 2 ', over which the particle-variables $\boldsymbol{a}$ and $\boldsymbol{b}$ run. There is only a single admissible pure state (and therefore there are no admissible mixed states), which is (the unit ray of) the celebrated anti-symmetric singlet-state:

$$
|\Psi\rangle \equiv \frac{1}{\sqrt{2}}\left(\left|z^{+}\right\rangle \otimes\left|z^{-}\right\rangle-\left|z^{-}\right\rangle \otimes\left|z^{+}\right\rangle\right) \in \mathbb{C}^{2} \otimes \mathbb{C}^{2} .
$$

Consider the following physically meaningful and permutation-symmetric 'Total-spin relation' (in units of $\hbar^{2} / 2$ ):

$$
\mathrm{T}(\boldsymbol{a}, \boldsymbol{b}) \text { iff } \quad\left(\sigma_{a}+\sigma_{b}\right)^{2}|\Psi\rangle=12|\Psi\rangle
$$

where

$$
\sigma_{1} \equiv\left(\sigma_{x}+\sigma_{y}+\sigma_{z}\right) \otimes 1 \text { and } \sigma_{2} \equiv 1 \otimes\left(\sigma_{x}+\sigma_{y}+\sigma_{z}\right)
$$

and where $\sigma_{x}, \sigma_{y}$ and $\sigma_{z}$ are the Pauli spin-matrices. Relation T (4) demonstrably discerns the two fermions weakly and categorically. ${ }^{15}$

Example 2 Consider a composite system of two arbitrary similar spinless particles and infinite-dimensional Hilbert-space $\mathcal{H}=L^{2}\left(\mathbb{R}^{3}\right)$. Let $\widehat{P}$ be the linear momentum operator and $\widehat{Q}$ the Cartesian position-operator acting in $L^{2}\left(\mathbb{R}^{3}\right)$. Consider the following physically meaningful and permutation-invariant 'commutator-relation':

$$
\mathrm{C}(\boldsymbol{a}, \boldsymbol{b}) \text { iff } \forall \Phi \in \mathcal{D}:\left[\widehat{P}_{a}, \widehat{Q}_{b}\right] \Phi=-\mathrm{i} \hbar \Phi,
$$

where $\mathcal{D} \subseteq L^{2}\left(\mathbb{R}^{3}\right) \otimes L^{2}\left(\mathbb{R}^{3}\right)$ is the domain of the commutator, and where

$$
\widehat{Q}_{1}=\widehat{Q} \otimes 1 \text { and } \widehat{Q}_{2}=1 \otimes \widehat{Q}
$$

and similarly for $\widehat{P}_{1}$ and $\widehat{P}_{2}$, respectively. Relation $C$ (6) discerns the two particles weakly and categorically. Extensions to pairs of particles of arbitrary spin by means of spinors proceed straightforwardly. ${ }^{16}$

We mention that the most of general proofs do neither rely on the projection postulate of QM nor on the standard property postulate of QM (a system has a quantitative property iff it is in an eigenstate of the corresponding operator), but they do

\footnotetext{
15 For the general proof for $N>1$ similar fermions in finite-dimensional Hibert-spaces, see Muller and Saunders (2008, pp. 535-536).

16 For the proof for $N>1$ similar particles for arbitrary spin in infinite-dimensional Hilbert-spaces, see Muller and Seevinck $(2009, \S 5)$.
} 
rely on the uncontroversial conjunct of the last-mentioned (if in an eigenstate, then a property).

In all discernibility-proofs, one begins with the numerical diversity of having $N>1$ named particles ( $N=2$ in the examples above). If one were to account for this numerical diversity by appealing to the names of the particles only, say, and not to anything physical about these particles, one would be spreading the smell circularity. But one does no such thing. The numerical diversity is accounted for physically, that is, by means of physically meaningful and permutation-invariant relations, as in the two examples above.

Let us now see how these results bear on OntSR.

\section{The second underdetermination thesis}

The French-Ladyman argument in favour of OntSR based on QM runs as follows. ${ }^{17}$ According to QM, an ontology of the truly and uniquely fundamental level of physical reality consisting of physical individuals must go, because similar elementary particles are indiscernible and indiscernibles are not individuals, they are not individual objects. Then, when you want to be realist with regard to QM (ScR), you better become a ontological structural realist (OntSR). But we have just seen that elementary particles are weakly discernible, their absolute indiscernibility notwithstanding. We have discernibles after all. This blocks the inference to OntSR for the scientific realist $(\mathrm{ScR})$ it seems.

More broadly speaking, consider the

Second Under-determination Thesis (2UT) Every scientific theory under-determines every specific metaphysical view of that part of the world the theory is about; in other words, every scientific theory has limited ontological content. ${ }^{18}$

The adjective 'specific' is to rule out trivial metaphysical views, such as 'There exists something', which presumably follows from every scientific theory and therefore is determined by it.

The on-going debates about the interpretation of QM provide a shining illustration of 2UT: a variety of distinct metaphysical views of the physical world can be consistently combined with QM. Let us next also consider a thesis opposite to 2UT, saying that scientific theories do have some ontological content:

Second Determination Thesis (2DT) Scientific theories are incompatible with some specific metaphysical views; in other words, they do have some definite ontological content.

\footnotetext{
17 Ladyman (1998); French and Ladyman (2003).

18 The under-determination of metaphysics by physics is a running theme in the philosophy of physics, e.g. $\S 6$ of French (2006). In harmony with this terminology, the familiar Duhem-Quine thesis about phenomena and data under-determining theory and model then has to be called 'the First Under-determination Thesis' (1UT).
} 
One illustration of 2DT we have provided in Sect. 1 on elementary particles: QM rules out the standard metaphysical view that accompanies classical physics, of space-time filled with interacting rigid bodies having worldlines. We also reported Schrödinger's conclusion, endorsed by many others, that QM also rules out every metaphysical view having elementary particles as individuals. This leaves us with an ontological dilemma between (i) metaphysical views of the world with genuine physical indiscernibles and (ii) metaphysical views with metaphysical individuals, which are metaphysically absolutely discernible objects, each having its own unique 'token-property' of haecceitas, or primitive thisness. ${ }^{19}$ If we count (i) and (ii) as different metaphysical views, then we have another illustration with QM of 2UT on our hands. The illustration of 2UT improves when we call to mind that we can also take elementary particles to be relationals, because this adds (iii) a metaphysical view with relationals. We then have an ontological trilemma in the face of QM, of which (i) and (ii) seem to point both to OntSR, but (iii) does not seem to do that. In this sense, then, OntSR seems under-determined by QM.

Nevertheless, there is a way to infer OntSR, even in the light of the fact that elementary particles are discernible relationals (iii). This way opens as soon as we call to mind that structures consist of objects with relations; these objects are determined only in so far as they are determined by the relations that constitute the structure. As quantum-mechanical objects, the elementary particles are determined by weakly discerning relations. Structuralist objects, if they must exist, should be relationals. Well, we have seen that is exactly what elementary particles demonstrably are: relationals. This leads us to OntSR. Whatever horn of the mentioned trilemma one chooses, one always ends up with OntSR. In this sense, 2UT fails. But there is more to be said.

The claim that elementary particles are relationals is not due to some interpretational gloss spread over the theory of QM, but is a theorem proved on the basis of only a few postulates of the theory of QM, and exactly the same holds for the claim that elementary particles are absolute indiscernibles. We are then permitted to deduce that the theory of QM determines (iii) and rules out (i) the view that elementary particles are physical indiscernibles. Since view (i) motivates (ii) — a metaphysical property is postulated in order to save the particles from indiscernibility-, view (ii) is left without motivation, because (i) has been confuted. We conclude that here 2UT fails because 2DT succeeds.

\footnotetext{
19 Sometimes 'self-identity' is taken to express haecceitas. Object $a$ has haecceitas, abbreviated by $h(a)$, iff $a=a$. But since the reflexivity of the identity-relation is an axiom of identity, every object described in a language that includes predicate logic (of course with identity) has haecceitas. Now it has become literally logically impossible for an object not to have haecceitas; its possession by everything we care to quantify over has become a logical necessity. To reject haecceitas, then, is to reject tautologies and to deny haecceitas is to float in contradictions. This is absurd. Hence 'self-identity' is not an acceptable expression of possessing haecceitas. Perhaps one could, when considering $N$ indiscernibles, introduce $N$ primitive monadic predicates in one's language, say $P_{1}, P_{2}, \ldots, P_{N}$, and then adopt the following axiom: a conjunction of $N$ conjuncts of the form: $j=k \longleftrightarrow P_{j}(k)$, where ' $j$ ' ranges over the predicate-subscripts and ' $k$ ' over the set of particles. This implies: $j \neq k \longleftrightarrow \neg P_{j}(k)$. Then we can define $h(k)$ as the disjunction $P_{1}(k) \vee P_{2}(k) \vee \cdots \vee P_{N}(k)$, so that every particle has haecceitas. Then every particle is metaphysically absolutely discernible. Denying haecceitas now has become a logical possibility: reject the mentioned axiom, or perhaps even better, adopt the following one: $\forall k, \forall j: \neg P_{j}(k)$.
} 
This raises, however, a pertinent question: are properties of elementary particles like mass, charge and spin-magnitude, determined by relations? This question needs to be answered in the affirmative by the structural realist (ScEpSR, OntSR), because the presence of properties in structures is permitted only when they are determined by relations. The answer is provided by the symmetry-group of QM, which is the Galilei-group.

The starting point of symmetry considerations is a relation between physical systems: one system being symmetric to another one, defined as there being a transformation (a map) that sends one to the other (active transformations); and a relation between descriptions of one physical system: one description being symmetric to another description of the same system, defined as there being a transformation (a map) that sends one description to another one (passive transformations). In physics the last-mentioned usually takes the form of an equation being covariant (form-invariant) under the transformation of the physical magnitudes occurring in the equation. Notice we then have three relations here (passing over the active symmetry transformation relation): an equation (which is a relation), the relation of covariance between equations, and physical magnitudes being related to transformed physical magnitudes. These transformations usually form a group. In QM the Galilei transformations form a connected Lie-group, which consist of the displacements and the rotations (they form the Euclidean group), and the Galilei-boosts (this can be extended by global phase-transformations of the state so as to obtain the Bargmann-group). The associated Lie-Algebra generates the Casimir-invariants, among which we find mass and spin-magnitude. Electric charge enters when we consider electromagnetic interactions. These properties of the physical system under consideration thus are determined by symmetry relations, which makes them acceptable for the structural realist.

Hence OntSR is the tailor-made version of scientific realism ( $\mathrm{ScR}$ ) for QM; then ScEpSR follows due to (1). Optimistic adherents of ScEpR will also be led to OntSR, due to (2). We conclude that the threat coming from the weak discernibility result has not only been allayed but it has been philosophically transformed into an argument in favour of OntSR. ${ }^{20}$

Acknowledgment Thanks to the Dutch National Science Foundation (NWO) for financial support and to remarks of an anonymous referee.

Open Access This article is distributed under the terms of the Creative Commons Attribution Noncommercial License which permits any noncommercial use, distribution, and reproduction in any medium, provided the original author(s) and source are credited.

\footnotetext{
${ }^{20}$ Until now we have been arguing with QM against a Galilean space-time background, which is not the space-time of our universe. We must move to Minkowskian space-time of the Special Theory of Relativity, and then to the semi-Riemannian space-time of the General Theory of Relativity. In fact relativistic quantum field theory — which is what one then ends up with — provides even better grounds for OntSR than QM. This will have to wait for another occasion.
} 


\section{References}

Castellani, E. (1998). Interpreting bodies. Classical and quantum objects in modern physics. Princeton, NJ: Princeton University Press.

Castellani, E., \& Mittelstaedt, P. (2000). Leibniz's principle, physics and the language of physics. Foundations of Physics, 30, 1587-1604.

Chakravartty, A. (2003). The structuralist conception of objects. Philosophy of Science, 70, 867-878.

French, S. (1989). Why the identity of indiscernibles is not contingently true either. Synthese, 78, 141-166.

French, S. (2006) Identity and individuality in quantum theory. In E. N. Zalta (Ed.), Stanford encyclopedia of philosophy. http://plato.stanford.edu/archives/spr2006/entries/qt-idind/.

French, S., \& Ladyman, J. (2003). Remodelling structural realism: Quantum physics and the metaphysics of structure. Synthese, 136, 31-56.

French, S., \& Redhead, M. L. G. (1988). Quantum physics and the identity of indiscernibles. British Journal for the Philosophy of Science, 39, 233-246.

Halvorson, H., \& Clifton, R. K. (2002). No place for particles in relativistic quantum field theory? Philosophy of Science, 69, 1-28.

Ladyman, J. (1998). What is structural realism? Studies in the History and Philosophy of Science, 29, 409-424.

Malament, D. B. (1992). In defense of Dogma: Why there cannot be a relativistic quantum mechanics of (localizable) particles. In R. K. Clifton (Ed.), Perspectives on quantum reality (pp. 1-10). Dordrecht: Kluwer.

Margenau, H. (1949). The exclusion principle and its philosophical importance. Philosophy of Science, 11, 187-208.

Monton, B., \& van Fraassen, B. C. (2003). Constructive empiricism and modal nominalism. British Journal for the Philosophy of Science, 54, 405-422.

Muller, F. A. (2005). The deep Black Sea: Observability and modality afloat. British Journal for the Philosophy of Science, 56, 61-99.

Muller, F. A., \& Saunders, S. W. (2009). Discerning all. British Journal for the Philosophy of Science, 59, 499-548.

Muller, F. A., \& Seevinck, M. P. (2009). Discerning all particles. Philosophy of Science (to appear).

Quine, W. v. O. (1976a). Grades of discriminability. Journal of Philosophy, 73(5), 113-116.

Quine, W. v. O. (1976b). Whither physical objects? In R. S. Cohen, et al. (Eds.), Essays in the memory of Imre Lakatos (pp. 497-504). Dordrecht: Reidel.

Redhead, M. L. G. (1995). More ado about nothing. Foundations of Physics, 25, 123-137.

Redhead, M. L. G., \& Teller, P. (1992). Quantum physics and the identity of indiscernibles. British Journal for the Philosophy of Science, 43, 201-218.

Saunders, S. (2006). Are quantum particles objects? Analysis, 66(1), 52-63.

Schrödinger, E. (1996). Nature and the Greeks and science and humanism. Cambridge: Cambridge University Press.

Weyl, H. (1928). Gruppentheorie und Quantenmechanik. Leipzig: Hirzel.

Worrall, J. (1989). Structural realism: The best of both worlds? Dialectica, 43, 99-124. 